\title{
Dietary guidelines to nourish humanity and the planet in the twenty-first century. A blueprint from Brazil
}

\author{
Carlos Augusto Monteiro 1,2,*, Geoffrey Cannon ${ }^{2}$, Jean-Claude Moubarac ${ }^{2}$, \\ Ana Paula Bortoletto Martins ${ }^{2}$, Carla Adriano Martins ${ }^{1,2}$, Josefa Garzillo², \\ Daniela Silva Canella ${ }^{2,3}$, Larissa Galastri Baraldi ${ }^{1,2}$, Maluh Barciotte $^{2}$, \\ Maria Laura da Costa Louzada ${ }^{1,2}$, Renata Bertazzi Levy ${ }^{2,4}$, Rafael Moreira Claro ${ }^{2,5}$ and \\ Patrícia Constante Jaime ${ }^{1,2}$ \\ 'Departamento de Nutrição, Faculdade de Saúde Pública, Universidade de São Paulo, Av. Dr Arnaldo 245, \\ São Paulo/SP, Brazil: ${ }^{2}$ Núcleo de Pesquisas Epidemiológicas em Nutrição e Saúde, Universidade de São Paulo, \\ São Paulo/SP, Brazil: ${ }^{3}$ Departamento de Nutrição Aplicada, Instituto de Nutrição, Universidade do Estado do Rio \\ de Janeiro, Rio de Janeiro/RJ, Brazil: ${ }^{4}$ Departamento de Medicina Preventiva, Faculdade de Medicina, \\ Universidade de São Paulo, São Paulo/SP, Brazil: ${ }^{5}$ Departamento de Nutrição, Universidade Federal de Minas \\ Gerais, Belo Horizonte/MG, Brazil
}

Submitted 17 April 2015: Final revision received 10 June 2015: Accepted 15 June 2015: First published online 24 July 2015

\begin{abstract}
Objective: To present and discuss the dietary guidelines issued by the Brazilian government in 2014.

Design: The present paper describes the aims of the guidelines, their shaping principles and the approach used in the development of recommendations. The main recommendations are outlined, their significance for the cultural, socioeconomic and environmental aspects of sustainability is discussed, and their application to other countries is considered.

Setting: Brazil in the twenty-first century.

Subjects: All people in Brazil, now and in future.

Results: The food- and meal-based Brazilian Dietary Guidelines address dietary patterns as a whole and so are different from nutrient-based guidelines, even those with some recommendations on specific foods or food groups. The guidelines are based on explicit principles. They take mental and emotional well-being into account, as well as physical health and disease prevention. They identify diet as having cultural, socio-economic and environmental as well as biological and behavioural dimensions. They emphasize the benefits of dietary patterns based on a variety of natural or minimally processed foods, mostly plants, and freshly prepared meals eaten in company, for health, well-being and all relevant aspects of sustainability, as well as the multiple negative effects of ready-to-consume ultra-processed food and drink products.

Conclusions: The guidelines' recommendations are designed to be sustainable personally, culturally, socially, economically and environmentally, and thus fit to face this century. They are for foods, meals and dietary patterns of types that are already established in Brazil, which can be adapted to suit the climate, terrain and customs of all countries.
\end{abstract}

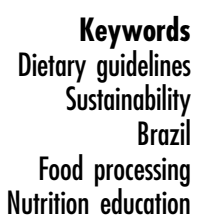

National dietary guidelines are official documents prepared and published by government agencies to guide public policy and to advise people on diets that promote health $^{(1)}$. Worldwide, more than fifty countries issue national dietary guidelines and an equal number is currently developing them ${ }^{(2)}$. However, most existing guidelines have a narrow view of how diet relates to health. In essence, they restrict the concept of diet to the amounts of nutrients contained in foods and the concept of health to the presence or absence of diseases caused by the lack or excess of one or more nutrients in diets.

The amount of nutrients in foods is of course relevant for health. But this is just one of the attributes of diets that are relevant to disease, health and well-being. The specific foods containing the nutrients are also relevant, because foods are not mere carriers of isolated nutrients ${ }^{(3-5)}$. Foods 
contain nutrients but also many other compounds with biological activity and potential to influence health, such as antioxidants and anti-inflammatory agents, and the synergistic combination of all these bioactive compounds affects how foods are digested, absorbed and metabolized $^{(3,6)}$. Also, foods are normally prepared, combined and consumed with other foods as dishes and meals and the nutrients and other bioactive compounds they contain all interact ${ }^{(3)}$. This explains why nutrients and other bioactive compounds separated from their food matrix, in the form of supplements or in 'fortified' processed food products, usually do not mimic their effects when consumed as a natural or intrinsic part of foods and of dishes and meals made with these foods ${ }^{(7-10)}$.

Other attributes of diets are usually understated in or even absent from current dietary guidelines. These include the contexts of eating, like when, why, where and with whom foods, dishes and meals are consumed. These circumstances all influence what kind of food is consumed and in what quantities, its digestion and absorption, and the pleasure in eating ${ }^{(11)}$.

Diet also includes the symbolic and emotional values of foods, dishes and meals embedded in all evolved societies. These values contribute to the enjoyment of eating, the building of memories and customs, and the strengthening of relationships and connections, which are aspects of health and well-being ${ }^{(12)}$.

Further, and particularly relevant for the present paper, foods are produced, transformed and supplied within food systems whose characteristics affect personal and population health and well-being through their impact on society and the environment. Food systems can be socially and environmentally sustainable, promoting justice and protection of the living and physical world; alternatively, they can generate many types of inequity and threats to natural resources and biodiversity ${ }^{(13)}$.

To summarize thus far, conventional dietary guidelines typically understate the relationship between diet and health. They treat foods as mere carriers of isolated nutrients, largely ignore food processing, meals and the contexts of eating, overlook the cultural dimensions of diets and do not consider the link between diet and the social and environmental sustainability of food systems.

Another limitation of conventional dietary guidelines is that they are slow to respond to changes in food supplies and patterns of population health and well-being. Current examples of these changes are the conversion of countless national and local food systems into one monolithic globalized industrial system dependent on intense food processing, the increasing availability of relatively cheap, aggressively marketed ready-to-consume soft drinks and snack products, and the parallel pandemic of obesity and diabetes ${ }^{(14,15)}$.

Dietary guidelines obviously need to be well-founded on evidence. But what is admissible as evidence and what is the best evidence? ${ }^{(16-18)}$ Most current dietary guidelines restrict their recommendations to quantities of nutrients, and sometimes of specific foods, believed to protect against specific diseases. This they do on the basis of knowledge provided by tightly controlled studies, of which randomized controlled trials are regarded the most valuable $^{(19)}$. Many other types of knowledge are usually not seen as relevant, one example being those implicit in long-established tried-and-tested dietary patterns developed as part of human evolution and adaptation ${ }^{(20,21)}$.

Further, rather than specifying rigid instructions, as they tend to do, dietary guidelines should empower people to be agents able to protect their own health and that of the people close to them, and to act in ways that affect social, environmental and other external determinants of health ${ }^{(22)}$.

The new official national Brazilian Dietary Guidelines, issued in November $2014^{(23)}$, aim to address and overcome common limitations of conventional dietary guidelines summarized above. Although many of these limitations have been acknowledged by UN agencies' reports since the $1990 \mathrm{~s}^{(1,24)}$, progress at national level has been slow. The present paper describes the new Brazilian Dietary Guidelines (hereafter simply called the guidelines'), ascertains how they overcome limitations, in particular those related to social and environmental sustainability of food systems and supplies, and considers their use internationally and in countries other than Brazil.

\section{Methods}

This section describes the aims of the guidelines, the process followed in their development, their shaping principles, and the approach used in the development of their recommendations.

\footnotetext{
Aims

The guidelines include a full set of information and recommendations about food, meals and eating practices whose purpose is to promote the health and well-being of people, families, communities and the whole Brazilian population, now and in future. They are for all Brazilians of all ages from 2 years, of all classes and from all regions. Target readers include health professionals, community workers, educators, capacity-building trainers and other professionals at all levels whose work includes health promotion. These and other workers are and will be crucial in the dissemination of the guidelines and to ensure that they are understood by all, including people who have difficulties in reading. The guidelines are planned to be used in people's homes, in health facilities, in schools and in all other places concerned with health and its promotion, such as community centres, social assistance reference centres, and in the work of civil society organizations and social movements.

While the guidelines are designed to promote health and well-being and to prevent illness, their recommendations may also help those affected by or suffering from
} 
specific diseases. In such cases dietitians will need to adapt the recommendations to the specific conditions of each person, supporting health professionals in the organization of nutritional care. Dietary guidance for children under the age of 2 years, consistent with the guidelines, is available elsewhere ${ }^{(25)}$.

\section{Process}

Preparation of the guidelines was a 3-year process directed by the Brazilian Ministry of Health with the technical assistance of the Centre for Epidemiological Studies on Health and Nutrition at the University of São Paulo, and support from the Pan American Health Organization. It involved two formal meetings with researchers, health professionals, educators and civil society representatives from all regions of Brazil, and regional meetings in the twenty-six Brazilian States and the Federal District. In early 2014, the draft guidelines were opened to public consultation. This resulted in 3125 responses from universities, public bodies, professional representative organizations, the private sector, health professionals and members of the public, enabling extensive revision of what has become a truly participatory national achievement.

The guidelines are an official publication of the Brazilian Ministry of Health. The document has 152 pages, is fully illustrated and so far is available online in three languages (English and Spanish, as well as the Portuguese version which is also printed). A first printing of 60000 copies has been distributed to health centres throughout the country. The guidelines have five chapters ('Principles', 'Choosing foods', 'From foods to meals', 'Modes of eating' and 'Understanding and overcoming obstacles'), a self-contained summary of the main recommendations as 'Ten Steps to Healthy Diets' and an annotated bibliography organized chapter by chapter.

\section{Principles}

We believe that the guidelines are the first to begin with an explicit set of principles. The first principle states that diet, as well as involving nutrients, is about foods, meals and eating modes, and also includes social and cultural aspects of dietary practices. The second states that sound dietary recommendations are tuned to respond to changes in food systems and supplies and patterns of population health. The third acknowledges the interdependence between healthy diets and the social and environmental sustainability of food systems. The fourth states that reliable recommendations on diet come from a range of sources of evidence. The fifth states that dietary guidelines should enlarge people's choice of and right to adequate and healthy diets (see Box 1).

\section{Development of recommendations}

The recommendations in the guidelines follow the principles set out above and concern choices of foods and meals, modes of eating and barriers to healthy diets.

\section{Choices of foods and meals}

The recommendations on food choices address four food groups defined by NOVA, the name for the classification developed at the University of São Paulo, which is based on the extent and purpose of industrial processing undergone by food before it is acquired, prepared and consumed $^{(26,27)}$.

The first NOVA group consists of natural or minimally processed foods. Natural foods are those obtained directly

Box 1 The five principles of the Brazilian Dietary Guidelines

1. Diet is more than intake of nutrients. Diet refers to intake of nutrients, and also to the foods that contain and provide nutrients. Diet also refers to how foods are combined and prepared in the form of meals, how these meals are eaten, and also to cultural and social dimensions of food choices, food preparation and modes of eating, all of which affect health and well-being.

2. Dietary recommendations need to be tuned to their times. Dietary recommendations should respond to changes in food supplies and in patterns of population health and well-being.

3. Healthy diets derive from socially and environmentally sustainable food systems. Dietary recommendations need to take into account the impact of the means of production and distribution of food on social justice and environmental integrity.

4. Different sources of knowledge inform sound dietary advice. Diet has various dimensions and a complex relationship with population health and well-being. Therefore, the evidence required to construct recommendations on diet is generated from different sources of knowledge.

5. Dietary guidelines broaden autonomy in food choices. Access to reliable information on characteristics and determinants of healthy diets contributes towards people, families and communities increasing their autonomy in making good food choices; it also contributes to leading them to demand compliance to the human right to adequate food. 
from plants or animals (such as vegetables and fruits, or eggs and milk) and acquired for consumption without having undergone any alteration following their removal from nature. Minimally processed foods are natural foods that have been submitted to cleaning, removal of inedible or unwanted parts, fractioning, grinding, drying, fermentation, pasteurization, cooling, freezing or other processes that do not add substances to the original food. The purpose of minimal processes is to preserve foods, to make it possible to store them, and sometimes also to simplify food preparation (cleaning and removing inedible parts), or to help their digestion, or make them more palatable (grinding or fermentation). Examples include grains that are dried, polished or ground as grits or flour; pasta made up from flour and water; roots, tubers and vegetables that are washed; dried fruits, frozen vegetables and fruits; cuts of meat that are cooled or frozen; and pasteurized milk.

The second NOVA group consists of processed culinary ingredients. These are substances extracted from natural foods or from nature itself by processes such as pressing, grinding, crushing, pulverizing and refining. The purpose of processing here is to produce ingredients used in homes and restaurants to season and cook natural or minimally processed foods and to make varied and enjoyable dishes such as soups, salads, dishes, grilled or roasted vegetables and meat, and handmade breads, pies, cakes and desserts. Examples of culinary ingredients are plant oils, lard, butter, sugar and salt.

The third NOVA group consists of processed foods. These are relatively simple food products manufactured essentially by the addition to natural or minimally processed foods of salt or sugar or other substance of common culinary use, such as oil or vinegar. The purpose of processing here is to prolong the duration of foods and enhance their palatability. Examples include canned and bottled vegetables or fruits; salted, smoked and cured meats and other foods; cheeses; and breads made of wheat flour, yeast, water and salt.

The ingredients and processes involved in the production of the items in the above three groups derive from the human cultural and technical experience and heritage beginning many thousands of years ago, while more recently being modernized in ways that can make those items more available, varied and safe. By sharp contrast, most of the products in the fourth NOVA food group, in their current forms, are novel industrial creations made possible by sophisticated modern technology including use of a myriad of food additives that give products hyperattractive colours, flavours, aromas and textures.

The fourth NOVA group consists of ultra-processed food and drink products. These are formulations mainly or solely of industrial ingredients. Their manufacture involves several stages and various processing techniques and ingredients, many used exclusively by industry. The purpose of processing here is to create durable, accessible, convenient and highly palatable ready-to-drink, readyto-eat or ready-to-heat products. These are typically consumed as snacks or desserts or as pre-prepared dishes and meals that displace natural or minimally processed foods and dishes and meals based on these foods and prepared from scratch. Examples include chips (crisps) and many other types of sweet, fatty or salty packaged snack products; ice cream, chocolate, candies; massproduced packaged breads, cookies, pastries, cakes; sweetened breakfast cereals; 'energy' bars; preserves; margarines; carbonated drinks, 'energy' drinks; sugared, sweetened milk drinks, sweetened including 'fruit' yoghurts and fruit drinks; cocoa drinks; infant formulas, follow-on milks, other baby products; 'health' and 'slimming' products such as powdered or 'fortified' meal and dish substitutes; and many ready-to-heat products including pre-prepared pies and pizza dishes, poultry and fish 'nuggets', burgers, hot dogs and other reconstituted meat products, and powdered and packaged soups, noodles and desserts.

Guidance on how to choose and combine foods as dishes and meals is based on evidence generated from analyses of data collected by the most recent Brazilian national dietary survey conducted in 2008-2009 ${ }^{(28,29)}$. These analyses have shown that diets made up from natural or minimally processed foods and dishes based on these foods and culinary ingredients are nutritionally far superior to diets made up from processed foods and ultraprocessed food and drink products. Such contrasts were most striking with nutrients whose consumption in the overall Brazilian diet is inadequate, such as dietary fibre and some vitamins and minerals, or excessive, such as sugar, saturated fats and trans fats. The survey analyses also have shown that the average diet of the one-fifth of the Brazilian population with the highest relative consumption of natural or minimally processed foods meets international recommendations for the consumption of most nutrients. These Brazilians represent the one-fifth (40 million people) of the total Brazilian population for whom natural or minimally processed foods and dishes and meals made from these foods amount to $85 \%$ or more of their total daily energy intake.

The recommendations on dishes and meals include photographs of examples of real breakfasts, lunches and dinners consumed by the one-fifth of Brazilians whose diet relies mostly on natural or minimally processed foods. These photographs show examples of meals of people of both genders, all age groups, all classes of income, from urban and rural settings, and from the five main regions of the country.

Guidance is also given on how to vary meals by substituting types of foods commonly consumed in Brazil with others of similar nutritional composition and culinary use, such as replacing beans with lentils or chickpeas, potatoes with cassava or yam, and okra with eggplant (aubergine) or pumpkin. Such variations, and their equivalents in other countries and cultures, enhance diversity in nutrients, 
flavours, aromas, colours and textures, and allow for regional, cultural and personal preferences.

The guidelines give no emphasis to the amount of foods or of dietary energy in each meal. This deliberate omission is because the nutritional and energy needs of people are very variable, depending on age, gender, size (weight and height) and level of physical activity. The control of body weight (and not the 'counting of calories') is suggested as a simple and efficient way to determine whether the amount of food being consumed is suitable.

\section{Modes of eating}

Recommendations on modes of eating address the circumstances - time, place, company, attention - that influence how foods are digested and absorbed, and also the pleasure of eating.

\section{Obstacles in the way of healthy diets}

Recommendations here focus on four main potential obstacles. These are: (i) the supply and cost of natural or minimally processed foods; (ii) the loss of culinary skills; (iii) lack of time; and (iv) the aggressive marketing of ultraprocessed products. Advice is given on how to surmount these obstacles, addressed to people as consumers, family and community members, and as citizens.

\section{Results}

This section describes the main recommendations of the guidelines on choices of foods and meals, on eating and on obstacles in the way of healthy diets.

\section{Recommendations on choices of foods and meals}

Box 2 shows the four recommendations and one golden rule for choices of foods and meals that make up nutritious, delicious and culturally appropriate diets and that also support and encourage socially and environmentally sustainable food systems.

As mentioned, recommendations on choices of foods and meals are illustrated by photographs of examples of actual breakfasts, lunches and dinners consumed by Brazilians whose diets, based on natural or minimally processed foods, approximate to the recommendations. These are followed by suggestions on how to multiply the examples of healthy meals.

Box 2 Choices of foods and meals

\section{Recommendations}

1. Make natural or minimally processed foods the basis of your diet. Natural or minimally processed foods, in great variety, mainly of plant origin, and preferably produced with agro-ecological methods, are the basis for diets that are nutritious, delicious, culturally appropriate and supportive of socially and environmentally sustainable food systems.

2. Use oils, fats, salt and sugar in small amounts for seasoning and cooking foods and to create culinary preparations. As long as they are used in moderation in culinary preparations based on natural or minimally processed foods, oils, fats, salt and sugar contribute towards diverse and delicious diets without rendering them nutritionally unbalanced.

3. Limit the use of processed foods, consuming them in small amounts as ingredients in culinary preparations or as part of meals based on natural or minimally processed foods. The ingredients and techniques used in the manufacture of processed foods - such as vegetables in brine, fruits in syrup, cheeses - unfavourably alter the nutritional composition of the foods from which they are derived.

4. Avoid ultra-processed products. Because of their ingredients, ultra-processed products - such as packaged snacks, soft drinks, sugared breakfast cereals, burgers and hot dogs (and as listed in the text above) - are nutritionally unbalanced. As a result of their formulation and presentation, they tend to be consumed in excess and displace natural or minimally processed foods and freshly made dishes and meals. Their means of production, distribution, marketing and consumption damage culture, social life, local economies and the environment.

\section{The golden rule}

Always prefer natural or minimally processed foods and freshly made dishes and meals to ultra-processed products. Opt for water, milk and fruits instead of soft drinks, dairy drinks and biscuits. Do not replace freshly prepared dishes (broth, soups, salads, sauces, rice and beans, pasta, steamed vegetables, pies) with products that do not require culinary preparation (packaged snacks and soups, instant noodles, pre-prepared frozen dishes, sandwiches, cold cuts and sausages, industrialized sauces, ready-mixes for cakes) and stick to homemade desserts, avoiding industrialized ones. 
Box 3 Modes of eating

1. Eat regularly and carefully. Always when possible, eat daily meals at similar times. Avoid snacking between meals. Eat slowly, with full attention, and enjoy eating without engaging in another activity.

2. Eat in appropriate environments. Always prefer to eat in clean, comfortable and quiet places, and where there is no stimulus to consume unlimited amounts of food.

3. Eat in company. Prefer eating with family, friends or colleagues. At home, share in the acquisition, preparation, cooking, and arrangements before and after eating.

\section{Recommendations on eating}

Box 3 presents the recommendations on the context of eating. Three aspects are considered: (i) the time and attention devoted to eating; (ii) the environment of eating; and (iii) the sharing of meals. When followed, the recommendations have various benefits. These include improved digestion and absorption of foods, more efficient control of what and how much food is consumed, enhanced family and social life, and more pleasure in eating.

The three recommendations on modes of eating are best followed all together. Regular and adequate times for meals require an appropriate environment, which is easier and more natural when people eat together. Appropriate environments help increase attention to eating. Eating in company discourages eating too quickly.

\section{Recommendations to overcome obstacles in the way of bealthy diets}

Because the recommendations of the guidelines are based on the actual diets of a substantial proportion of Brazilian families, they are realistic and feasible. They are also flexible. They do not include instructions on amounts of foods or nutrients. Instead, terms like 'prefer' and 'most of the time' are used. There are practically endless combinations and amounts of foods that make up healthy diets.

Nevertheless, following all the recommendations will not always be easy. As mentioned, the main potential obstacles include the supply and actual or perceived cost of natural or minimally processed foods; loss of culinary skills; lack of time; and aggressive marketing of ultraprocessed products. Overcoming these obstacles is likely to involve considerable commitment, patience and persistence, and some cannot be overcome personally. It is helpful always to bear in mind the value of nourishing diets for good health and well-being, and also the fact that illness caused by unhealthy diets and ways of life cost time and money as well as causing misery. It is well worthwhile to pay more attention to food and eating. This often implies making new decisions about what is most important in life.

Box 4 is of recommendations designed to overcome obstacles in the way of healthy diets. These address people personally. They also address people as citizens, who as well as voting, can work to protect what is good and to change what is bad, locally, municipally and nationally. This is essential for adoption of enlightened public policies and effective regulatory actions that will benefit whole populations. People can act as citizens themselves, as members of neighbourhood, community, work and school groups, as users of public services, and also as members of political parties, civil society organizations or social movements.

In Brazil there are many civil society groups dedicated to the protection and strengthening of public health. Citizens can join and be part of their work. This includes pressing for public policies, including regulations and support systems that can make the country an environment that enables the recommendations of the guidelines.

\section{Discussion}

The Brazilian guidelines are, we believe, the first to fully incorporate the need for sustainability. This we do not see as an optional 'extra', but as essential in considering the value and significance of diet in the context of dietary patterns, and food systems and supplies, not merely now, but also for the future. All dietary guidelines should work well at the time of issue and also, modified as knowledge grows and circumstances change, for people who are children now and also for those not yet born.

The focus of the guidelines on sustainability is illustrated in Fig. 1. The guidelines are designed to nourish and sustain the bodies of the people of Brazil (the lower segment of the diagram with some examples of benefits) and also their minds and souls (the upper-left segment). Their purpose is also to protect and preserve the planet and the biosphere (the upper-right segment). These three aims are indivisible parts of the whole of sustainability in the full sense of the concept, including all aspects of well-being of the human species in the living and physical environment and the biosphere.

All the recommendations in the guidelines have sustainability in mind. Here we focus on three recommendations and their significance for the cultural, social, economic and environmental aspects of sustainability.

\section{Recommendation: Make natural or minimally processed foods the basis of your diet}

Natural or minimally processed foods, in great variety, mainly of plant origin, and preferably produced 
Box 4 Overcoming obstacles in the way of healthy diets

1. Be mindful when shopping for food and eating out of home. Avoid places that sell or serve mainly or only ultraprocessed foods. In supermarkets take and use a shopping list. Support farmers' markets, municipal markets, specialist retailers and other places that sell varieties of natural and minimally processed foods, and prefer food produced by ecological methods. When you can, grow some of your own food, even if only herbs. Join groups to buy healthy food in bulk. Be aware that many staple minimally processed foods are cheap. Prefer vegetables and fruits that are in season and are locally produced. When eating out, choose self-service restaurants that charge by weight. Press municipal authorities to install public facilities that sell natural or minimally processed foods at affordable prices. Campaign for policies that support local food farmers and growers and that protect the prices of their produce.

2. Develop, exercise and share culinary skills. If you have culinary skills, develop them and share them, especially with boys and girls. If you do not have these skills - men as well as women - acquire them. Learn from and talk with people who know how to cook. Ask family, friends and colleagues for recipes, read books, check the Internet and eventually take courses. Start cooking. Press for the inclusion of cooking and other culinary skills as part of formal school curriculum. Engage with civil society associations committed to promotion of the Brazilian cultural heritage, gastronomy, and regional and local cuisines.

3. Plan your time to make food and eating important in your life. Plan the food shopping, organize your domestic stores and decide on meals in advance. Simple everyday meals do not take much time. Share with family members the responsibility for all activities related to meals. Make the preparation and eating of meals privileged times of conviviality and pleasure. Assess how you live so as to give proper time for food and eating. Appreciate that exchanging time taken for home entertainment like watching television, for time together before and after meals and at table, brings deeper satisfaction and improves family life, especially for children.

4. Be wary of food marketing. Be critical of all forms of food marketing. Explain to children and young people that the essential purpose of advertising is to increase product sales and not to inform or educate people. Study the Brazilian legislation, which protects consumer rights, and denounce those who do not follow it.

with agro-ecological methods, are the basis for diets that are nutritious, delicious, culturally appropriate and supportive of socially and environmentally sustainable food systems.

Worldwide, plentiful varieties of natural or minimally processed foods of plant origin combined with small quantities of foods of animal origin, eaten as such and as contained in dishes and meals, result in nutritious, delicious and appropriate diets ${ }^{(30)}$. They also entail and imply food systems and supplies that are relatively equitable, and less stressful to the physical environment, for animals and biodiversity in general $^{(30)}$.

Different varieties of plant foods such as grains, legumes, roots and tubers, vegetables and fruits, when consumed as natural or minimally processed foods, are mostly produced by family farmers in Brazil ${ }^{(31)}$ and in most countries ${ }^{(32)}$. Therefore, consumption of such foods also ensures and strengthens local economies, and living and working in solidarity. It also promotes biodiversity and reduces the environmental impact of food production and distribution ${ }^{(32-36)}$.

Reduced consumption and thus production of animal foods in general will reduce emissions of greenhouse gases that are a cause of climate disruption, will reduce deforestation caused by creation of industrial-scale farms to grow crops for animal feed and to graze the animals themselves, and also will reduce the use of water ${ }^{(36)}$. Further, it will reduce the number of intensive animal production systems. The crowding characteristic of these systems stresses the animals, concentrates animal wastes, requires systematic use of antimicrobial drugs known to generate bacteria resistant to drugs for human use, pollutes and contaminates groundwater, reservoirs, lakes and rivers, and generates diseases of animals that transmit to people ${ }^{(37,38)}$.

Intensive animal production also requires vast amounts of animal feed produced by monocultures such as of soyabean and corn. Like all modern industrial agriculture, these also require prodigal use of water and of toxic biocides, herbicides, pesticides and fertilizers that contaminate sources of water, degrade soil, increase pest resistance and reduce biodiversity ${ }^{(39)}$. The use of genetically modified seeds is also a cause for environmental concern in Brazil ${ }^{(40)}$ and elsewhere ${ }^{(41,42)}$.

Food systems based on agro-ecological methods produce foods free of chemical contaminants, protect biodiversity, promote more diverse diets, contribute to fair distribution of productive lands and the creation of work, and respect and improve knowledge and traditional forms of production. The more that people see the benefits of sustainably produced foods and prefer them, the greater will be the support received by farmers who respect the land and the more socially and environmentally sustainable food systems will become ${ }^{(43-46)}$. 


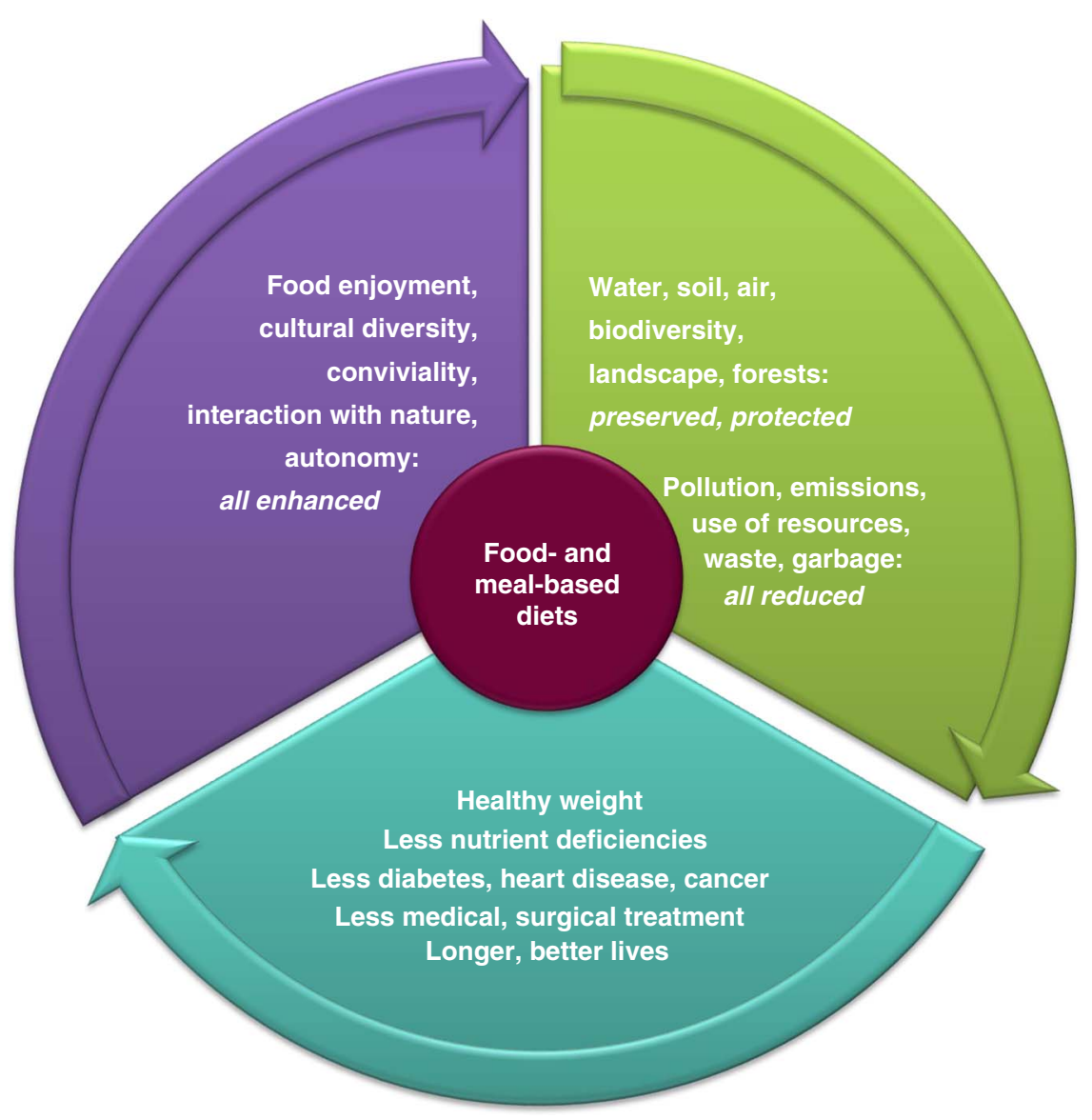

Fig. 1 Aims of the Brazilian Dietary Guidelines

\section{Recommendation: Avoid ultra-processed products}

Because of their ingredients, ultra-processed products - such as packaged snacks, soft drinks, sugared breakfast cereals, burgers and hot dogs (and as listed in the text above) - are nutritionally unbalanced. As a result of their formulation and presentation, they tend to be consumed in excess and displace natural or minimally processed foods and freshly made dishes and meals. Their means of production, distribution, marketing and consumption damage culture, social life, local economies and the environment.

There are many reasons to avoid the consumption of ultra-processed food and drink products, including their commonly excessive energy density and gross nutrient imbalances. These reasons are specified and discussed elsewhere ${ }^{(27,28,47-51)}$. Ultra-processed products, produced and consumed in the amounts now characteristic in modern industrialized countries and settings, also have damaging impacts on culture, social life, local economies and the environment, as seen below.

\section{Culture}

The advertising, promotion, branding, packaging, labelling and contents of brands of ultra-processed products tend to be or become identical throughout the world. A brand of soft drink made by one giant manufacturer is essentially the same the world over. Types of burger made by various manufacturers are much the same everywhere. Leading brands are promoted often using the same entertainers, models, music and slogans everywhere, including on television, the Internet and social media. They are disseminated by means of intensive and aggressive advertising campaigns, including the launching of a vast number of new products every year, creating a false sense of diversity. Such campaigns, which may be linked with billion-dollar multi-media entertainment packages beamed at children and young people, displace and invalidate traditional foods and eating patterns, culinary knowledge and skills, and whole social cultures ${ }^{(14,51)}$.

All this pushes a sense especially to children and young people that the culture and identity of their own country, region, ethnicity and tradition are uninteresting. Major ultra-processed product manufacturers in effect act in concert, because they have the same overall aim, to displace authentic and infinitely rich and varied cultures 
and replace these by a monolithic uniform consumer culture whose driving force is more and more consumption and profit ${ }^{(14,51)}$.

\section{Social life}

Ultra-processed products are formulated and packaged to be ready-to-consume with limited or no preparation. This makes meals and sharing of food at the table unnecessary. Ultra-processed products can be consumed anytime, anywhere, often when being entertained or when working, or when walking in a street, driving or talking on a phone. These are mostly isolated situations, disguised by advertisements suggesting that such products promote social interaction, which they do not ${ }^{(14,51)}$.

\section{Local economies}

The demand for cheap oils, sugar, starches and other common ingredients of ultra-processed products promotes crop monocultures and industrialized farms throughout the world that produce raw materials including for export and not foods for direct human consumption. This damages and can even destroy local food systems, traditional knowledge of appropriate agronomy and rural employment, family and community life, and skills needed to live well. It also contributes to food and nutrition insecurity and thus alienation and dependence on foreign food aid and food supplementation programmes ${ }^{(14,51)}$.

\section{Environment}

The impact of ultra-processed products on the physical environment is also damaging. Intensive monocultural production of their main ingredients is dependent on systematic use of biocides and fertilizers and excessive use of water. The manufacture and distribution of most ultra-processed products involve long transport routes and thus more excessive use of non-renewable energy and water, and emission of pollutants. This all causes environmental degradation and pollution, loss of biodiversity and depletion and loss of water, energy and other natural resources. Their production and consumption also cause creation of vast amounts of waste and garbage, dumped in disgusting and dangerous landfill sites. Overall, ultraprocessed products, with many interrelated factors, threaten the environment and the biosphere ${ }^{(14,51)}$.

\section{Recommendation: Eat in company}

Prefer eating with family, friends or colleagues. At home, share in the acquisition, preparation, cooking, and arrangements before and after eating.

Human beings are social creatures. Eating together is ingrained in human history, as is the sharing and division of responsibility for finding, acquiring, preparing and cooking food. Eating together, with everything involved with eating, is part of the evolution and adaptation of the human species and the development of culture and civilization. Eating together is a natural, simple yet profound way to create and develop relationships between people ${ }^{(21)}$.

The sharing of meals at home is a precious and important time for family members and others who live together to cultivate and strengthen their social and emotional ties. Meals are important moments to teach children and adolescents about sharing and respect and connecting with others ${ }^{(52)}$.

Eating in company when away from home, including at work or school, helps colleagues and friends to get acquainted and to exchange experiences. Occasions such as weddings, birthdays, and other celebrations marked by special meals, are memorable times for people who like one other and want to be together.

Thankfully, it is still common to share meals in Brazil, as it still is in very many other countries. Daily meals are usually prepared for the whole family. Friends from work or school prefer to eat in company. Shared meals at weekends and as celebrations retain their special value. This tradition is a precious part of national culture, varied regionally and in different States, which needs to be protected and strengthened. All the more so because all over the world, food cultures based on shared meals are being undermined by the increasing manufacture, promotion and consumption of ultra-processed products ${ }^{(53-56)}$.

\section{Universal value}

In a personal communication to us, Olivier de Schutter, former UN special rapporteur on the right to food, writes:

I made the Brazilian Guia the central message of my final address as UN Special Rapporteur. Now we must work towards encouraging other countries and regions to seek inspiration from the Guia in their own nutritional policies.

\section{Brazil as an example}

Brazil is unusual in various ways. It is a vast country of 200 million people, with an area roughly half that of Latin America, or of the USA minus Alaska. It is sometimes said that 'all human life is in Brazil', from the cosmopolitan centres of its great cities and its rapidly growing middle classes in big and smaller cities and towns, to cooperative and family farming communities, to impoverished populations living in favelas and in communities originally founded by escaped slaves, and the original Brazilian people, some still secluded in Amazonia.

Brazil also has retained its own rich food cultures in its five main regions, each bigger than most European countries. Many traditional foods, dishes and balanced meals, originated in many countries including Brazil and appropriately adapted over generations to suit climate, terrain and custom, are special to Brazil, as of course is still the case in many other countries ${ }^{(14)}$. Plus Brazil is formally constituted and actually organized as a participatory 
democracy with a resilient and resourceful system of civil society organizations and social movements, whose representatives have helped to shape the guidelines.

\section{Facing this century}

All this said, we believe that the guidelines, and their rationale and approach outlined here, may apply to other countries, now and for the foreseeable future.

We all of us human beings are now living in a unique time in history. This century is a turning time for life on Earth. There is no escaping this fact. To contribute to the continued welfare and existence of human society and civilizations, all relevant sciences and other activities in principle and in action must now be sustainable, and prove that they are sustainable. All of us who have some responsibility for shaping food systems and supplies and dietary patterns must be part of this great endeavour.

We all have to face the facts of our times now and of this century. These include human-made climate disruption; precipitate urbanization; the pollution, degradation and depletion of air, land, water and sources of energy; and the outrageous abuses and waste caused by mass production of animals ${ }^{(57)}$.

These also include food and nutrition insecurity; the emergence of a global system that has made food, like money, a commodity subject to speculation that jeopardizes established varied rational, equitable and economical national and regional food systems everywhere; gross and widening inequities between and within countries; and a rocketing world population ${ }^{(50,58,59)}$.

\section{Sustainability is essential}

Therefore, the recommendations of dietary guidelines everywhere now must be planned to be indefinitely sustainable in all respects: personally, culturally, socially, economically, politically and environmentally. Public health nutrition policies need to be in line with all other policies affecting the human, living and physical world and the biosphere. Dietary guidelines and the concepts on which they are based that are not tuned to our times now and as reliably projected need to be reviewed.

In many other countries in all continents, national and local food systems and supplies survive, along with food cultures based on minimally processed foods and freshly prepared meals eaten in company ${ }^{(60-62)}$. For these countries, like Brazil, the way ahead is clear. As outlined here and in the guidelines, the task of protecting, maintaining and strengthening these systems, supplies and cultures, with all that is entailed, is not and will not be easy. But with foresight, we believe it is possible.

In some high-income countries where most dietary energy consumed is already in the form of branded ultraprocessed products ${ }^{(47,63)}$ and where regular everyday meals are increasingly uncommon ${ }^{(64)}$, the task will be most difficult.
It will also be very difficult in those countries including in Africa whose external debt burdens force their governments to give special access and privileges to transnational ultra-processed product corporations, and where farmers are pressed to grow cash crops for export rather than food for their own communities ${ }^{(65-69)}$.

However hard, dangerous and steep the road ahead, we see no other rational way. What is needed, with food and nutrition, just as with climate disruption ${ }^{(70,71)}$, is radical. Tinkering will not work and can only make bad worse. Thus, the desperate policy of reformulating ultraprocessed products so that, somewhat less unhealthy, they can make health claims that further distract attention away from genuinely healthy foods, consolidates the crisis $^{(50,72)}$.

\section{Our gift}

There are beacons. Once, few people believed that breastfeeding could be revived, or that smoking could be controlled and reduced. But in many countries, including Brazil, public actions to promote breast-feeding and to control smoking are succeeding ${ }^{(73,74)}$.

Any problem once recognized is halfway to its solution. We believe that the Brazilian Dietary Guidelines face the facts of this century. Our work in recent years ${ }^{(14,26,27,48,50,51,53,63,72,75-77)}$ and its practical application in our own national guidelines are offered for friends and colleagues in other countries and continents, to adapt to their own circumstances, customs and cultures.

\section{Acknowledgements}

Acknowledgements: We thank our colleagues Denise Coutinho, Enrique Jacoby, Francine Lima and Semíramis Martins Álvares Domene, who are part of the team that formulated the Dietary Guidelines for the Brazilian Population. We also thank the General Coordination of Food and Nutrition (Ministry of Health) and the Brazilian office of the Pan-American Health Organization for the collaboration and support, and Regina Rodrigues for the administrative assistance. Financial support: This research received no specific grant from any funding agency in the public, commercial or not-for-profit sectors. Conflict of interest: None. Authorship: All authors participated in the development of the guidelines and of the paper. C.A.M., G.C. and J.-C.M. drafted the paper, with substantial contributions from the other authors. All authors approved the final version of the paper.

\section{References}

1. Food and Agriculture Organization of the United Nations/ World Health Organization (1998) Preparation and Use of Food-Based Dietary Guidelines. WHO Technical Report Series no. 880. Geneva: FAO. 
2. Food and Agriculture Organization of the United Nations (2015) Food-based dietary guidelines website. http://www.fao. org/nutrition/nutrition-education/food-dietary-guidelines/en/ (accessed July 2015).

3. Jacobs DR \& Tapsell LC (2013) Food synergy: the key to a healthy diet. Proc Nutr Soc 72, 200-206.

4. Fardet A \& Rock E (2014) Toward a new philosophy of preventive nutrition: from a reductionist to a holistic paradigm to improve nutritional recommendations. Adv Nutr $\mathbf{5}$, 430-446.

5. Scrinis G (2013) Nutritionism: The Science and Politics of Dietary Advice. New York: Columbia University Press.

6. Fardet A (2015) A shift toward a new holistic paradigm will help to preserve and better process grain products' food structure for improving their health effects. Food Funct $\mathbf{6}$, 363-382.

7. Jacobs DR, Pereira MA, Meyer KA et al. (2000) Fiber from whole grains, but not refined grains, is inversely associated with all-cause mortality in older women: the Iowa women's health study. J Am Coll Nutr 19, 3 Suppl., S326-S330.

8. Miller ER 3rd, Pastor-Barriuso R, Dalal D et al. (2005) Meta-analysis: high-dosage vitamin E supplementation may increase all-cause mortality. Ann Intern Med 142, 37-46.

9. Omenn GS, Goodman GE, Thornquist MD et al. (1996) Effects of a combination of $\beta$ carotene and vitamin $\mathrm{A}$ on lung cancer and cardiovascular disease. N Engl J Med 334 , $1150-1155$.

10. Klein EA, Thompson IM Jr, Tangen CM et al. (2011) Vitamin E and the risk of prostate cancer: the selenium and vitamin $\mathrm{E}$ cancer prevention trial (SELECT). JAMA 306, 1549-1556.

11. Cohen D \& Farley TA (2008) Eating as an automatic behavior. Prev Chronic Dis 5, A23.

12. Mintz S \& Du Bois C (2002) The anthropology of food and eating. Annu Rev Anthropol 31, 99-119.

13. Food and Agriculture Organization of the United Nations (2010) Sustainable Diets and Biodiversity: Directions and Solutions for Policy, Research and Action. Proceedings of the International Scientific Symposium Biodiversity and Sustainable Diets United Against Hunger. Rome: FAO.

14. Monteiro CA \& Cannon G (2012) The impact of transnational 'big food' companies on the South: a view from Brazil. PLoS Med 9, e1001252.

15. Pan American Health Organization (2014) Consumption of Ultra-Processed Food and Drink Products in Latin America: Trends, Impact on Obesity, and Policy Implications. Washington, DC: PAHO.

16. Margetts B, Warm D, Yngve A et al. (2001) Developing an evidence-based approach to Public Health Nutrition: translating evidence into policy. Public Health Nutr 4, 1393-1397.

17. Kroke A, Boeing H, Rossnagel K et al. (2004) History of the concept of 'levels of evidence' and their current status in relation to primary prevention through lifestyle interventions. Public Health Nutr 7, 279-284.

18. World Cancer Research Fund/American Institute for Cancer Research (2007) Food, Nutrition, Physical Activity, and the Prevention of Cancer: A Global Perspective. Washington, DC: WCRF/AICR.

19. US Departament of Agriculture \& US Department of Health and Human Services (2010) Dietary Guidelines for Americans, 2010, 7th ed. Washington, DC: US Government Printing Office.

20. Kuhnlein HV (2014) Food system sustainability for health and well-being of Indigenous Peoples. Public Health Nutr (Epublication ahead of print version).

21. Wrangham R (2009) Catching Fire: How Cooking Made Us Human. New York: Basic Books.

22. Freire P (2013) Education for Critical Consciousness. New York: Bloomsbury Academic (reprint edition).

23. Ministry of Health of Brazil (2014) Dietary Guidelines for the Brazilian Population. Brasília: Ministério da Saúde.
24. World Health Organization (1999) Development of FoodBased Dietary Guidelines for the Western Pacific Region. Manila: WHO Western Pacific Region Office.

25. Ministry of Health of Brazil (2010) Ten Steps for a Healthy Feeding: Feeding Guide for Child Under Two Years: A Guide for the Professional in the Primary Health Care. Brasília: Ministério da Saúde.

26. Monteiro CA, Cannon G, Levy RB et al. (2014) Ultraprocessing and a new classification of foods. In Introduction to US Food System Public Health, Environment, and Equity, pp. 338-339 [R Neff, editor]. San Francisco, CA: Jossey-Bass.

27. Moubarac J-C, Parra D, Cannon G et al. (2014) Food classification systems based on food processing: significance and implications for policies and actions. A systematic literature review and assessment. Curr Obes Rep 3, 256-272.

28. Louzada MLC, Martins AP, Canella D et al. (2015) Ultraprocessed foods and the nutritional dietary profile in Brazil. Rev Saude Publica (Epublication ahead of print version).

29. Louzada MLC, Martins AP, Canella D et al. (2015) Impact of ultra-processed foods on micronutrient content in the Brazilian diet. Rev Saude Publica (In the Press).

30. Katz D \& Meller S (2014) Can we say what diet is best for health? Annu Rev Public Health 35, 83-103.

31. Instituto Brasileiro de Geografia e Estatística (2009) Censo Agropecuário 2006: Agricultura Familiar - Primeiros Resultados. Brasil, Grandes Regiões e Unidades da Federação. Rio de Janeiro: IBGE.

32. Food and Agriculture Organization of the United Nations (2014) The State of Food and Agriculture 2014. Innovation in Family Farming. Rome: FAO.

33. Rockström J, Steffen W, Noone K et al. (2009) Planetary boundaries: exploring the safe operating space for humanity. Ecol Soc 14, 1.

34. Gerber P, Steinfeld H, Henderson B et al. (2013) Tackling Climate Change Through Livestock. A Global Assessment of Emissions and Mitigation Opportunities. Rome: FAO.

35. Jalava M, Kummu M, Porkka M et al. (2014) Diet change - a solution to reduce water use? Environ Res Lett 9, 1-14.

36. Tilman D \& Clark M (2014) Global diets link environmental sustainability and human health. Nature 515, 518-522.

37. Food and Agriculture Organization of the United Nations (2006) Livestock's Long Shadow: Environmental Issues and Options. Rome: FAO

38. Food and Agriculture Organization of the United Nations (2011) World Livestock 2011: Livestock in Food Security. Rome: FAO.

39. West PC, Gerber JS, Engstrom PM et al. (2014) Leverage points for improving global food security and the environment. Science 345, 325-328.

40. Furnival AC \& Pinheiro SM (2008) A percepção pública da informação sobre os potenciais riscos dos transgênicos na cadeia alimentar. Hist Cienc Saude Manguinhos $\mathbf{1 5}$, 277-290

41. Macilwain C (2010) World view: talking the talk. Nature 465, 867.

42. Romero H (2015) (2015) EU passes controversial GMO food Law. Deutsche Welle, 13 January. http://www.dw.de/eupasses-controversial-gmo-food-law/a-18188282 (accessed April 2015)

43. Wezel A, Bellon S, Doré T et al. (2009) Agroecology as a science, a movement and a practice. A review. Agron Sustain Dev 29, 503-515.

44. Chappell MJ \& LaValle LA (2011) Food security and biodiversity: can we have both? An agroecological analysis. Agric Hum Values 28, 3-26.

45. De Schutter O (2014) Report of the Special Rapporteur on the Right to Food. Geneva: UN Human Rights Council.

46. DeClerck FA, Fanzo J, Palm C et al. (2011) Ecological approaches to human nutrition. Food Nutr Bull 32, 1 Suppl., S41-S50. 
47. Moubarac JC, Martins AP, Claro RM et al. (2012) Consumption of ultra-processed foods and likely impact on human health. Evidence from Canada. Public Health Nutr 16, 2240-2248.

48. Monteiro CA, Levy RB, Claro RM et al. (2011) Increasing consumption of ultra-processed foods and likely impact on human health: evidence from Brazil. Public Health Nutr 14, $5-13$.

49. Ludwig DS (2011) Technology, diet, and the burden of chronic disease. JAMA 305, 1352-1353.

50. Moodie R, Stuckler D, Monteiro C et al. (2013) Profits and pandemics: prevention of harmful effects of tobacco, alcohol, and ultra-processed food and drink industries. Lancet 381, 670-679.

51. Monteiro CA, Cannon G, Levy RB et al. (2012) The food system. Ultra-processing. The big issue for nutrition, disease, health, well-being. World Nutr 3, 527-569.

52. Simmons D \& Chapman G (2012) The significance of home cooking within families. Br Food J 114, 1184-1195.

53. Martins AP, Levy RB, Claro RM et al. (2013) Increased contribution of ultra-processed food products in the Brazilian diet (1987-2009). Rev Saude Publica 47, 656-665.

54. Moubarac JC, Batal M, Martins AP et al. (2014) Processed and ultra-processed food products: consumption trends in Canada from 1938 to 2011. Can J Diet Pract Res 75, 15-21.

55. Crovetto M \& Uauy R (2012) Changes in processed food expenditure in the population of Metropolitan Santiago in the last twenty years. Rev Med Chil 140, 305-312 (in Spanish).

56. Juul F \& Hemmingsson E (2015) Trends in consumption of ultra-processed foods and obesity in Sweden between 1960 and 2010. Public Health Nutr (Epublication ahead of print version).

57. McMichael AJ (2013) Globalization, climate change, and human health. $N$ Engl J Med 368, 1335-1343.

58. Stuckler D \& Nestle M (2012) Big Food, food systems, and global health. PLoS Med 9, e1001242.

59. Ercsey-Ravasz M, Toroczkai Z, Lakner Z et al. (2012) Complexity of the international agro-food trade network and its impact on food safety. PLoS One 7, e37810.

60. Trichopoulou A \& Lagiou P (1997) Healthy traditional Mediterranean diet: an expression of culture, history, and lifestyle. Nutr Rev 55, 383-389.

61. Lee MJ, Popkin BM \& Kim S (2002) The unique aspects of the nutrition transition in South Korea: the retention of healthful elements in their traditional diet. Public Health Nutr 5, 197-203.

62. Bere E \& Brug J (2009) Towards health-promoting and environmentally friendly regional diets - a Nordic example. Public Health Nutr 12, 91-96.

63. Moubarac JC, Claro RM, Baraldi LG et al. (2013) International differences in cost and consumption of ready-toconsume food and drink products: United Kingdom and Brazil, 2008-2009. Glob Public Health 8, 845-856.

64. Schlosser E (2001) Fast Food Nation. London: Penguin Books.

65. Gonzalez C (2006) Markets, monocultures, and malnutrition: agricultural trade policy through an environmental justice lens. Michigan State J Int Law 14, 345-382.

66. National Research Council (2006) Lost Crops of Africa. vol. I: Grains. Washington, DC: National Academy Press.

67. National Research Council (2006) Lost Crops of Africa. vol. II: Vegetables. Washington, DC: National Academy Press.

68. National Research Council (2008) Lost Crops of Africa. vol. III: Fruits. Washington, DC: National Academy Press.

69. Redclift M (1987) Sustainable Development: Exploring the Contradictions. London: Methuen.

70. Stern N (2009) The Global Deal. Climate Change and the Creation of a New Era of Progress and Prosperity. New York: Public Affairs.

71. Klein N (2014) This Changes Everything. Capitalism versus the Climate. New York: Simon and Schuster.

72. Monteiro CA \& Cannon G (2014) The food system. Product reformulation will not improve public health. World Nutr $\mathbf{5}$, 140-158.

73. Venancio SI, Saldiva SR \& Monteiro CA (2013) Secular trends in breastfeeding in Brazil. Rev Saude Publica 47, 1205-1208.

74. Monteiro CA, Cavalcante TM, Moura EC et al. (2007) Population-based evidence of a strong decline in the prevalence of smokers in Brazil (1989-2003). Bull World Health Organ 85, 527-534.

75. Monteiro CA, Moubarac JC, Cannon G et al. (2013) Ultraprocessed products are becoming dominant in the global food system. Obes Rev 14, Suppl. 2, S21-S28.

76. Monteiro CA, Gomes FS \& Cannon G (2010) The snack attack. Am J Public Health 100, 975-981.

77. Canella DS, Levy RB, Martins AP et al. (2014) Ultraprocessed food products and obesity in Brazilian households (2008-2009). PLoS One 9, e92752. 